\title{
The Oral Mucosa and Langerhans Cells in Smokers: Evidence for Carcinogenesis
}

Khoo SP, Lee KW. The Oral Mucosa and Langerhans Cells in Smokers: Evidence for Carcinogenesis. Annals Dent. Univ. Malaya 1995;2:1-4.

\begin{abstract}
A study was carried out to investigate whether smoking had any effect on the Langerhans cells in the oral mucosa, which might throw light onto the mechanism of malignant transformation of some keratotic lesions in the oral cavity. Thirty-two cases of keratotic lesions from biopsy specimens of smokers and non-smokers were studied. Langerhans cells were identified by immunocytochemical staining for $\$ 100$ proteins and their densities quantified. Smokers were associated with a significant reduction in the Langerhans cell population compared to non-smokers. The mean values of Langerhans cell density in light smokers and heavy smokers were $28.64 / \mathrm{mm}^{2}$ and $33.421 \mathrm{~mm}^{2}$ respectively compared to $66.51 / \mathrm{mm}^{2}$ in nonsmokers. There was a dose-response relation between the number of cigarettes smoked daily and the effect on cell counts. These findings of a local immunological effect of smoking on oral epithelium may explain the means by which cigarette smoking contributes to the development of oral cancer.
\end{abstract}

Key Words: Smoking, Langerhans cells, oral epithelium, malignant transformation

\section{INTRODUCTION}

Smoking has been identihed as a risk factor in the development of oral cancer $(1,2)$ and cervical neoplasia $(3,4)$.The mechanism by which this occurs, however, is unclear. Smoking has been shown to have an adverse effect on several aspects of the immune response $(5,6)$ It has been postulated that localised depletion in Langerhans cell (LC) numbers cause an impairment in the local immune response, thus predisposing the tissue to malignant change (6).

Langerhans cells are dendritic epithelial cells that originate from the bone marrow and are important in presenting antigens to $\mathrm{T}$ cells. In common with other cells of the monocyte/macrophage lineage, they express Fg-lgG receptors, the Class 11 Major Histocompatibility Antigen HLA DR, HLA DQ and HLA DR (7,8). S100 protein is also another marker expressed by LCs (9).

This study was undertaken to investigate whether smoking is associated with a depletion in the local LC population, a change that might suggest that epithelial structural changes are accompanied by a functional decrement such as been suggested to occur as a prelude to the onset of cervical neoplasia.

\section{MATERIALS AND METHOD}

Archival material from 32 individuals were selected for the purpose of this study. The cases were specimens from biopsies taken from patients who presented with keratotic lesions in the oral cavity. They comprise of 14 males and 18 females with ages ranging from 25 to 75 years and were categorized into 3 major groups ie. nonsmokers (NS), light smokers (LS) and heavy smokers
(HS). The consumption of more than 20 cigarettes per day was used as a criterion in differentiating a HS from a LS. Only cigarette and cigar smokers were selected to the exclusion of other forms of tobacco habit. The amount smoked per day was recorded in each case. Table 1 shows the distribution of age, sex and tobacco comsumption in the three sample groups.

Table 1 Distribution of age, sex \& tobacco consumption in the three sample groups

\begin{tabular}{|c|c|c|c|c|}
\hline \multirow{2}{*}{$\begin{array}{l}\text { Group } \\
\text { NS (9) }\end{array}$} & \multicolumn{2}{|c|}{ Sex (M/F) } & \multirow{2}{*}{$\frac{\text { Avg. age } \pm S D}{63 \pm 11}$} & \multirow{2}{*}{$\frac{\text { Avg. Cig./day } \pm \text { SD }}{0}$} \\
\hline & 3 & 6 & & \\
\hline LS(12) & 6 & 6 & $51 \pm 14$ & $16 \pm 5$ \\
\hline HS(11) & 5 & 6 & $50 \pm 12$ & $49 \pm 35$ \\
\hline
\end{tabular}

Sample size in parentheses; $\mathrm{Avg}=$ average; SD = standard deviation

The keratotic lesions consists of sublingual keratosis, smokers' keratosis/nondiagnostic keratosis, lichenoid leukoplakia and frictional keratosis. The lesions were obtained from all regions of the oral mucosae but generally they were classified under keratinized and non-keratinized epithelium (Table 2)

Table 2 Distribution of keratinization amongst data groups

\begin{tabular}{cccc}
\hline \multirow{2}{*}{ Group } & \multicolumn{3}{c}{ Type of keratinization } \\
& PK & OK & Mixed \\
\hline NS & 2 & 2 & 5 \\
LS & 4 & 2 & 6 \\
HS & 2 & 3 & 6 \\
\hline Total & 8 & 7 & 17 \\
\hline
\end{tabular}

$\mathrm{Pk}=$ parakeratosis $; \mathrm{OK}=$ orthokeratosis 
The specimens were fixed in $10 \%$ formal saline and embedded in parafin wax. Three sequential 4 um thick sections were cut from each case; for staining with haematoxylin and eosin (H \& E), anti S100 polyclonal antibodies using PAP technique and Masson Fontana. The details of the anti S100 PAP technique are described elsewhere (10).

Two controls were carried out for S100, one without the primary antibody and the other without the secondary antibody. Two positive controls were also carried out using a skin specimen and sections from a neuroma. Positive controls on skin specimens were carried out for Masson Fontana stains. The S100 positive cell densities and Masson Fontana positive cell densities were measured and the difference revealed the LC densities.

\section{LC Quantification:}

The cell densities were obtained with the aid of a semi-automatic analysis system 'IBAS 1' (Kontron) which was calibrated before any measurements actually began. A software (Videoplan Program) operating the system computes the various geometric parameters and data. An objective lens (X40) was used to obtain a sharp and large image of the cells and epithelium and also to reduce the human error in tracing. A randomized systemic sampling was carried out where the test fields were placed at equal intervals across the section guided by a rectangular frame displayed on the monitor screen. This ensures no overlap of test fields and yields small errors (11).

Only cells showing a positive cell body with at least one associated dendrite were counted. Cell counts were followed by measurements of epithelial area in order to obtain parameters for cell densities.

The data obtained was transferred into an IBM compatible computer in a anumerical Database format. A one-way analysis of variance (ANOVA) on a statistical program (STATGRAPHICS) was used to compare the LC density among data groups (A 5\% level of significance was adopted).

\section{RESULTS}

Langerhans cells were observed to be distributed in both the basal and the lower prickle layers in the three sample groups. In NS, the LCs were large with prominent cell bodies and distinct nuclei. There were more dendritic processes and were clearly defined. In smokers, however, LCs were smaller with ill-defined dendritic processes. Furthermore these processes were shorter and fewer in number and in some cases were absent altogether. There was no obvious difference between the LS and HS groups. Figures 1 and 2 illustrate the findings.

The distribution of LC density is shown in Table 3.

Table 3 Distribution of LC density $\left(\mathrm{pt} / \mathrm{mm}^{2}\right)$

\begin{tabular}{cc}
\hline Group & Avg. \pm SD \\
\hline NS & $66.5 \pm 40.38$ \\
LS & $28.64 \pm 25.60$ \\
HS & $33.42 \pm 25.79$ \\
\hline
\end{tabular}

Avg. = average $; \mathrm{SD}=$ standard deviation
There is a threefold decrease in LC density in the LS group while that in the HS group is less than threefold when compared to NS.

One-way analysis of variance of the findings and the least significant difference test (LSD) showed that only the LC density in the NS group vary significantly from the rest as indicated by the $95 \%$ confidence interval and a significant level of $\mathrm{p}=0.0201$ (Table 4). The LC densities in the HS and LS groups however did not

Table 4 Least Significance Diference test to determine group homogeneity

\begin{tabular}{ccccc}
\hline Level & Average & SE & $\begin{array}{c}95 \% \text { Confidence } \\
\text { groups }\end{array}$ & Homogeneous \\
\hline NS & 66.51 & 13.45 & $51.83-81.19$ & $* *$ \\
LS & 28.64 & 7.39 & $15.92-41.36$ & $*$ \\
HS & 33.41 & 7.77 & $20.13-46.69$ & $*$ \\
\hline
\end{tabular}

$\mathrm{PE}=$ standard error

show any significant difference. These findings clearly show that LC density tend to decrease among smokers. A Sample Correlation Analysis with a value of $\mathrm{r}=-0.17$ clearly demonstrates an inverse relationship between the number of cigarettes smoked per day and LC density.

In general most of the lesions were of the mixed keratinization type. Hyperkeratosis was more prominent in NS and LS as compared to HS (Figs. 3 and 4). There



Figure 1. Langerhans cells as appear in a non-smoker showing large cell bodies with numerous long dendritic processes (S100 PAP X 1033)

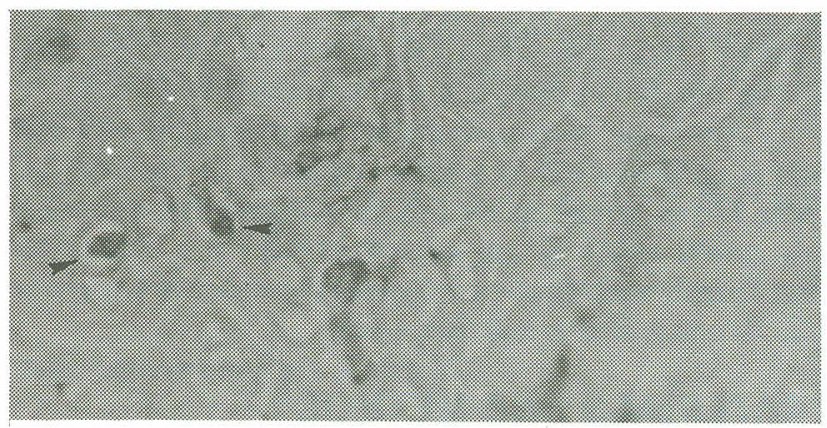

Figure 2. Langerhans cells in a heavy smoker exhibiting smaller cell bodies with fewer and shorter dendritic processes (left arrow). Processes may be absent in some instances (right arrow). (S100 PAP X 1033)

were more cases of acanthotic epithelium in HS than in either of the other two groups. The types of keratinization were not particularly associated with atrophic or acanthotic epithelium. Cellular atypia did not appear to be a prominent feature. Table 2 summarizes the 




Figure 3. Histopathological features of a smokers' keratosis lesion in a light smoker. The squamous epithelium is hyperorthokeratinized and hyperplastic but cellular atypia is not present in this case. A prominent granular layer is also seen ( $H$ \& E X 413)

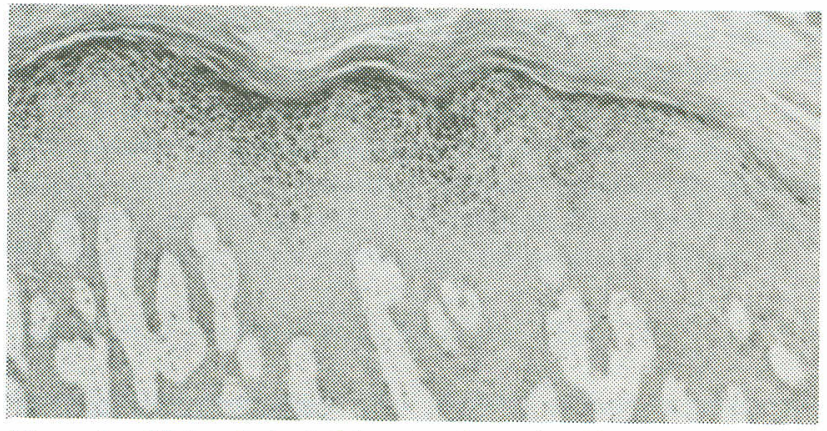

Figure 4. Histopathological features of a smokers' keratosis lesion in a heary-smoker. The squamous epithelium is hyperparakeratotic and acanthotic. Minimal cellular atypia is present but not seen under this magnification (NB. epithelium is less keratotic than in light smoker) $(H$ $\mathcal{E} E X 413)$

distribution of the types of keratinization and the presence of cellular atypia in the data groups.

\section{DISCUSSION}

The reduction in LC densities in keratotic lesions in smokers as shown in this study concur with previous observations that mucosal or cutaneous lesions that may develop into cancer are associated with a significant reduction in $\operatorname{LCs}(6,12,13)$. In vivo experimental carcinogenesis have suggested that. loss of LCs during tumour promotion stage may impair immunologic protection against skin tumours (14).

The reduction in LC densities could represent the death of LCs due to irritating or toxic agents in tobacco smoke. The death could occur within the epithelium or within the connective tissue. In the latter, the tobacco constituents act like a chemotactic agent when present in the circulation, attracting LCs towards the connective tissue, hence its depleted counts within the epithelium. The presence of small molecular weight components in smoke extracts (possible haptens) can react with other proteins and serve as immunogens capable of stimulating an immune response which might ultimately be cytotoxic to LCs(15). In lichen planus, for example, it has been hypothesised that LCs and macrophage process and present antigens to T-cells. Presumably after a proliferative period, T8 Iymphocytes, a T-cell subset, become cytotoxic for basal keratinocytes(16). Therefore it is interesting to speculate that such a mechanism could operate here too.

Very few studies have actually shown that a reduction in cell numbers has occured $(12,17)$. Possibilities other than cell death which account for the reduced LC densities, include the reduction of S100 protein production probably due to antigenic transformation in some cells. The transformation could be attributed to ultrastructural changes such as swollen mitochondria, membrane disruption, cytolysis or loss of dendritic processes in LCs following exposure to contact allergens in murines (18). A quantification study run concurrently with a qualitative ultrastructural study would help to set this perspective right.

The reduction in numbers of LCs have also been observed in smokeless tobaccoassociated lesions has been postulated by some workers, to be a result of changes caused by smokeless tobacco and is one factor in the long-term pathogenesis of smokeless-tobaccoassociated oral carcinoma (12). Other possible cofactors include the presence of human papillomavirus, which has been demonstrated in cervical intraepithelial neoplasia (19), alcohol, which has been shown to act locally to promote carcinogenesis (20). Barrett and workers (21) have demonstrated the interaction between alcohol and tobacco and they suggested that there is possibly a systemic influence of alcohol in combining with the direct effect of tobacco to elicit a response in the LC population.

Studies have observed a dose-response relationship between the number of cigarretes smoked daily and the reduction in LC counts in clinically normal and lesional epithelium of the cervix $(6,22)$ and lesional epithelium of the oral mucosa (12). The amounts of reduction noted by these authors were similar to those noted in lesional specimens in this study. This inverse relationship implies that the progressive reduction in the number of LCs available to detect and present antigens to T-cells may facilitate the persistence of antigens which in tum could increase the risk of neoplastic transformation.

In considering the effects of smoking on the epithelium as a whole, it was demonstrated that hyperkeratinization occured in most of the cases especially in the NS and LS groups. The lesser degree of keratinization seen in most cases of HS could be attributed to the nature of the keratinocytes. In LS and NS, minimal agitation of its cells might produce large amounts of keratinization as a protective measure. On the other hand in HS, the large amount of assault might produce hyperplasia rather than hyperkeratinization.

The particular type of keratinization present were, however, not associated with atrophic or acanthotic epithelium. These histological findings were not entirely in agreement with those of Pindborg et al (23) in which they related epithelial changes to various tobacco habits. They observed that the predominant epithelial change in cigarrette smokers was acanthosis associated with hyperkeratinized epithelium while in bidi-smokers atrophy was associated with hyperorthokeratinization. The histology of cigarrete smokers in the present study did not show such a relationship. There were no explanation offered in Pindborg's study to substantiate their observation. The dfflerence in observations between these two studies is likely to be due to the subjective methods used in assessing the epithelial thickness.

- The reduction in LC numbers offers some possible 
explanation as to why smokers rather than non-smokers have an increased risk of developing oral cancer. The tobacco smoke may reduce the number of LCs available to detect and present antigens to T-cells thus rendering the epithelium vulnerable to toxicarcinogenic substances, for example, even to the tobacco smoked subsequently. This depletion results in anergy of the immune system which will then fail to check the development of any transformed cells. This has also been hypothesised by Barton and workers (6), that tobacco compromises the local immune defences in female smokers by causing a reduction in the number of local LCs thus rendering these patients susceptible to cervical neoplasia.

The major health concern regarding smoking is its association with premalignant oral epithelial changes $(1,2)$ and the increased risk of uterine cervical cancer (22). Therefore there is a need for further investigation into the etiology and pathogenesis of oral cancer. In such a study a highly selective group of patients is required. In this study, only cigarette and cigar smokers were considered as it has been shown that the effects of pipe-smoking are less prominent in the lungs and oral cavity than in the systemic circulation whereas cigarette smoke tend to stay longer in the oral circulation so exerting a prominent effect there (24).

This study serves as a pilot for another similar but larger study which is being carried out using more sensitive techniques. The limitations in this study have been taken into considerations in the interpretation of the results in this study.

In summary, this study suggests that tobacco smoking affects LCs which in turn may result in anergy of the immune system thus increasing the risk of malignant transformation.

\section{REFERENCES}

1. Pindborg JJ, Mehta FS, Gupta PC, Daftery DK, Smith CJ. Reverse smoking in Andhra Pradesh, India. A study of palatal lesions among 169 villagers. Br J Cancer 1971, 25: 10-20,

2. Kahn HA. The Dom study of smoking and mortality among US veterans: report on eight and one half years of observation. Natl Cancer Inst Monogr 1966, 19: 1 -125

3. Wigle DT, Grace M. Smoking and cancer of the uterine cervix. Am J Epidemiol 1980, 111: 125-7

4. Trevathan E, Layde P, Webster LA, Adams JB, Benigo BB, Ory H. CigareHe and smoking and dysplasia and carcinoma-in-situ of the uterine cervix. JAMA 1983, 250: 499-502

5. Miller LG, Goldstein G, Murphy M, Ginns LC. Reversible alterations in immunoregulatory T cells in smoking. Chest 1982, 5: 527-9

6. Barton SE, Maddox PH, Jenkins J, Edwards R, Cuzick J, Singer A. Effect of smoking on cervical epithelial immunity: a mechanism for neoplastic change? Lancet 1988, 2: 6524,

7. Stingl G, Katz Sl, Clement L, Green 1, Shevach EM. Immunilogical function of la-bearing epidermal Langerhans cells. J Immunol 1978, 121: 2005-13

8. Sontheimer RD, Stasny P, Nunez G. HLA-D region antigen expression by human Langerhans cells. J Invest Dermatol 1986, 87: 707-10

9. Cocchia D, Michetti F and Donato R. Immunochemiocal and immunocytochemical localization of S100 antigen in normal human skin. Nature 1981, 294: 85-87

10. Charbit Y, Monteil RA, Hitzig C, Sauget P, Bendiche N, Jasmin JR. S100 immunolabelling of Langerhans cells in oral epithelium. J Oral Pathol 1986, 15: 419-422,

11. Tawfik AM, El-Labban and Lee KW. Alteration in stereological density parameters of the mucosal vascular bed during neoplastic transformation in sublingual keratosis. Path Res Pract 1989, 184: 519-524

12. Daniels TE, Chou L, Greenspan JS, Grady DG, Hauck WW, Greene JC, Emster VL. Reduction of Langerhans cells in smokeless tobacco-associated oral mucosal lesions. J Oral Pathol Med 1992, 21: 100-4

13. Pitigala-Arachchi A, Crane IJ, Scully C, Prime SS. Epithelial dendritic cells in pathological human oral tissues. J Oral Pathol Med 1989, 18: 11-6

14. Halliday GM, Odling KA, Ruby JC, Muller HK. Suppressor cell activation and enhanced skin allograft survival after tumor promoter but not initiator induced depletion of cutaneous Langerhans cells. J Invest Dermatol 1988, 90: 293-7

15. Lehrer SB, Stankus RP, Selvaggio JE. Tobacco smoke sensitivity: A result of allergy? Ann Allergy 1986, 56: 369-380

16. Bjerke J. Subpopulations of mononuclear cells in lesions of psoriasis, lichen planus and discoid lupus erythematosus studied using monoclonal antibodies. Acta Derm (Stokh) 1982, 62: 477-483

17. Tosca A, Varelzidis A, Michalopuolos M and Strtigos J: In situ identification of mononuclearcells in lichen planus. Dermatologica 1983, 167: 113-120

18. Picut CA, Lee CS, Lewis RM. Ultrastructural and phenotypic changes in Langerhans cells induced in vitro by contact allergens. Br J Dermatol 1987, 116: 773-784

19. Viac J, Guerin-Reverchon 1, Chardonnet Y, Bremond A. Langerhans cells and epithelial cell modifications in cervical intraepithelial neoplasia: cerrelation with human papillomavirus infection. Immunobiology 1990,180: 328-38

20. Blott WJ, McLaughlin JK, Winn DM et al. Smoking and drinking in relation to oral and pharyngeal cancer Res 1988, 48: 3282-7

21. Barrett AW, Williams DM, Scott J. Effect of tobacco and alcohol consumption on the Langerhans cell population of human lingual epithelium determined using a monoclonal antibody against HLADR. J Oral Pathol Med 1991, 20: 49-52

22. Winkelstein W. Smoking and cervical cancer - current status: a review. Am J Epidemiol 1990, 131: 945-57

23. Pindborg JJ, Srivasta AN and Gupta D. Studies in oral leukoplakia Vlll. Epithelial changes in tobacco-induced leukoplakia in India. Acta Odontol Scand 1964, 22: 499-512,

24. Doll R and Peto R. The causes of cancer. 1981, 1st edition. Oxford University Press 